\title{
Detecting Digital Tampering by Blur Estimation
}

\author{
Dun-Yu Hsiao, Soo-Chang Pei \\ Department of Electrical Engineering, \\ National Taiwan University \\ silverseraph@gmail.com_pei@cc.ee.ntu.edu.tw
}

\begin{abstract}
With powerful computer and mighty software, seasoned users could turn digital media into what they want. The detection of digital tampering has become a crucial problem. In most of the time, digital tampering is not perceptible by human; however, some traces of digital tampering may be left in the media during the process. Based on this idea, we present a method in this paper that could reveal blurred regions that indicate possible tampering without any embedded information such as watermarking technique. Effectiveness and results will be presented, robustness would also be discussed.
\end{abstract}

\section{Introduction}

Considering the making of digital image frauds, for example, face replacement, in order to produce a seamless doctored face, applying blurring is inevitable. Other than face replacement, skin smoothing and panorama are also blurring involved digital image tampering. Blurring is a very common process in digital image manipulation; it could be used to reduce the degree of discontinuity or to remove unwanted defects, ultimately, it is used to generate plausible digital image forensics. Hence if an additional blurring process applied on an image is detectable, possible fraud can be exposed even in a credible image.

We will first introduce our blur estimation method. From the fundamentals of frequency domain knowledge about the mathematical model, we develop a scheme to detect blur regions for general images then modify it for identifying digital forensics.

To illustrate the general effectiveness of this technique, we show results on naturally blurred images first to prove the effectiveness of our blur estimation method and proceed on perceptually credible forgeries applied manually smoothing, and then demonstrate the possibility to detect computer-generated objects in movie scenes by our blur estimation scheme.

\section{Review on digital forgeries detection}

A study about how to verify digitally altered contents without the implementation of watermark becomes a novel and emerging subject. However, several researchers have carried out some representing results in different approaches, but these approaches are different from ours. 
A method that could be able to detect duplicate regions, which are produced by copy and paste operations, is proposed by Fridrich et al. [15]. Chang et al. [11-14] introduce a passive-blind approach, by bicoherence, without prior knowledge as embedded information to detect spliced images. Farid et al. [15] have proposed several useful techniques to expose digital image forgeries such as the features of higher-order correlations in the frequency domain for natural signals, and the trace of image re-sampling. Farid et al. have more works about this domain, such as the study about detecting digital tampering by an EM-based color filter interpolation approach. However, for more details, we urge readers to the references [4-10].

Different from above prior studies, our test images are collected from the Internet. We believed that if we want to achieve a more practical method for digital image forgery detection, we have to use real-life images to test.

\section{Blur estimation}

In the making of digital image frauds, considering the face replacement process, imaginable, the size of the two faces are not likely to match, in most of the time the author would commence a resizing procedure. Resizing will cause the two regions become similar in their dimension, then the author have to adjust the angle that the face towards by rotating it. Moreover, the dissimilarity of the joining regions, intensity or color, which is resulted from light source direction (such as from left or right, makes the shadows have non-consistent positions) or type (a tungsten light or fluorescent tube, which will cause identical object exhibits different colors), adjusting brightness or contrast will preserve global illumination consistency, and correcting color can remove biased color effect resulted from different light source, adding or erasing shades would gain shading homogeneity.

Till now, the forged region is quite similar to the original, but it won't be exactly identical to the covered one; consequently, removing the seamy regions is ineluctable. To achieve this goal, a ubiquitous way is to apply a Gaussian blur on the seamy areas. After that, a believable face replacement fraud is done, such as Fig. 1: the face of the center image is cropped, resized, rotated, re-lighted, smoothed and pasted onto the left one to obtain the result image.
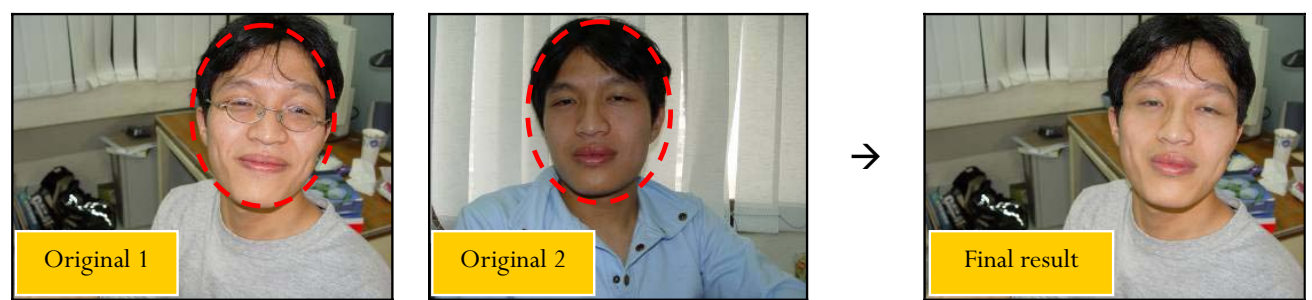

Figure 1. Another face replacement artifact made by us, above two images are the original inputs. The result image has been resized, rotated and applied illumination correction then smoothed to conceal edges. The lighting effect isn't very accurate in our fraud image.

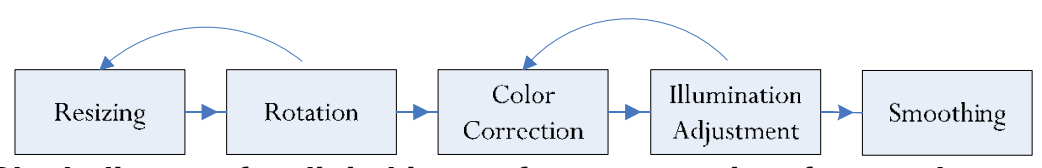

Figure 2. Block diagram for digital image forgery creation, face replacement.

In all procedures a face replacement encompasses, which are shown in Fig. 2, the most 
essential element is blurring: in order to remove unwanted boundaries, in order to make a seamless and plausible replaced face, applying blurring is indispensable and inevitable. If you create a face replacement tampering without erasing the seams, you will fail to make a believable forgery.

Since blurring process is necessary, if we could trace such a process we could exposed the existence of digital image tampering. In order to trace blurring process, we should know-what a blur look like and how it occurs in an image. The answers are quite simple, there are two major kinds blur in digital images: defocus blur and manually low pass filtering (or Gaussian blur), just like Fig. 3 shows. When it comes to digital tampering, it often refers the latter.
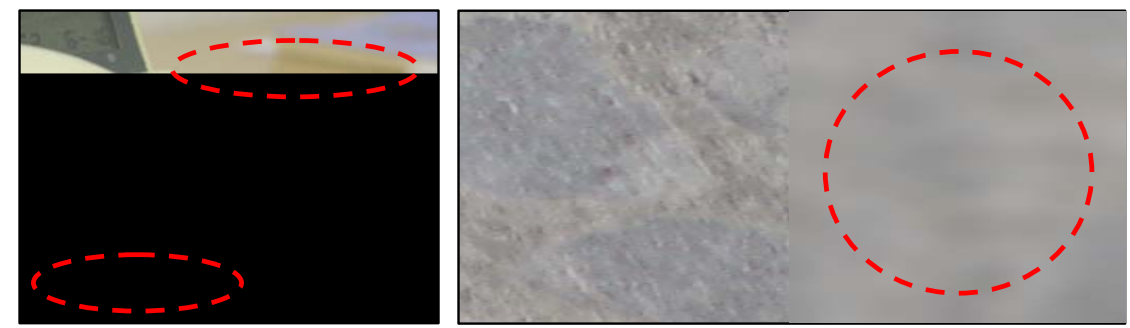

Figure 3. Left, defocus blurred image, and right, manually blurred image.

Based on the prior knowledge of a blurred signal, we propose a new blur estimation scheme with a beneficial feature, that is, only a single image is required. This is important to digital image forensics detection because it is often that we could acquire the tampered subject only.

There are plenty of former studies about how to perform image enhancement or blur removal on the defocused region to restore the content [1-3], hence these propositions have the abilities to detect, locate, or estimate the blurred regions of images. However, most of these algorithms require iterative operations, complex blur models or many image sources-which are the restrictions that cripple themselves even when encountering a usual problem-to acquire a result. First of all, a procedure similar to Marichal's [1] is applied, which uses DCT domain knowledge to estimate a rough quality of the entire image by calculating and summarizing the histogram of DCT coefficients. Next, normalizing and taking a threshold on each DCT blocks of the image are performed. Some metrics are defined and calculate the difference to distinguish blurred regions from sharp ones. The last phase is to estimate the degree of blurring by combining the prior knowledge and the distance information. After those operations, the blurred regions are predicted and presented. Detailed blur estimation method based on frequency domain knowledge is described below.

In the beginning of blur estimation, we have to clarify what a blur is; such a clarification requires a definition of edge. Here it is assumed that an edge is an integral of Gaussian signal as Equation (1) shows; this is a common assumption that usually used to represent edge signal. Because it's a Gaussian integral, by adjusting $\sigma_{b}$, it could shape various kind of edge, steep or moderate.

$$
\int_{-\infty}^{t}\left(1 / \sqrt{2 \pi} \sigma_{b}\right) \cdot e^{-\frac{x^{2}}{2 \sigma_{b}^{2}}} d x
$$

As for blur model, it is usually simplified as a Gaussian blur filter illustrated as Equation (2).

$$
\left(1 / \sqrt{2 \pi} \sigma_{b}\right) \cdot e^{-\frac{x^{2}}{2 \sigma_{b}^{2}}}
$$

Again, by varying the term $\sigma_{b}$, it is easy to obtain different sizes of Gaussian signals. Notice that the Gaussian signal is symmetric and the Fourier transform of Gaussian signal is still in a form of Gaussian. 


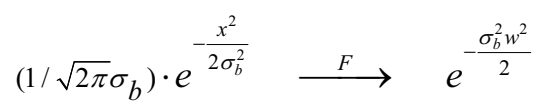

Furthermore, the image capture system is modeled as follows.

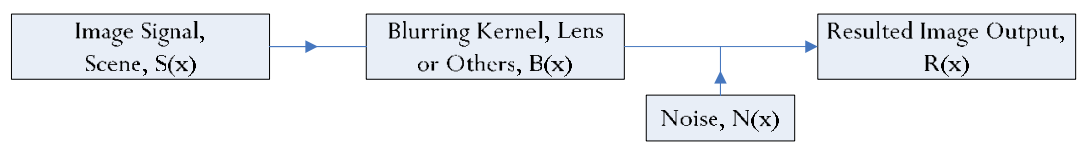

Figure 4. The block diagram of image capture system model.

The input signal $S(x)$, which is the light emitted from the composition of the scene, blurred by the lens function $B(x)$ (low-pass filtering, assumed as Gaussian blur, which is a symmetric function), accompanied with noise $N(x)$ (such as thermal noise produced from CCD), result in the output image $R(x)$. With the signal and system models, try considering the case of $R(x)-S(x)$, and what if $R(x)-S(x) \sim 0$ ?

$$
\begin{gathered}
B(x) \cdot S(x)-S(x)=e^{-\frac{\sigma_{b}^{2} w^{2}}{2}} \cdot S(x)-S(x) \\
S(x) \cdot B(x)-S(x) \sim 0
\end{gathered}
$$

Because $B(x)$ is symmetric, if the input signal is a gradual ramp function, the difference between $S(x) \cdot B(x)$ and $S(x)$ will be very small. On the other hand, the region with sharp edges would exhibit great difference between above two terms. This phenomena is very reasonable because the blur function will preserve low frequencies, when the variance is large, which means there are a greater portion of high frequency terms, thus a clearer edge will be shown.

In most cases, the addition noise is relative smaller than the real signal, if this term is omitted; Equation (5) will be rewrite as:

$$
R(x)-S(x) \sim 0
$$

Deducing from above equation, we assume the perfect edge and perfect blur are

$$
\left\{\begin{array}{lll}
\arg \max (R(x)-S(x)), & \text { perfectly } & \text { sharp } \\
\arg \min (R(x)-S(x)), & \text { perfectly blurry }
\end{array}\right.
$$

Note that the Fourier transform of the Gaussian is still in a form similar to Gaussian, Gaussian function will suppress the high frequencies of the power spectrum; hence for a signal, or a certain kind of texture, their power spectrums before and after blurring would look like those in Fig. 5 (a) (b). Although the figures are not drawn to scale, which means in the power spectrum of a sharp signal A, there may exhibit a larger intensity of low frequency components than those in a blurry signal B, for example, a greater DC term in a sharp signal spectrum than in a blurred one; however, the figures are here to express the different in spectrum distribution between the two signals. What is more, if we restrict the spectrum for only a certain kind of signal (or texture), the relationships (sharp and blurred ones) will match the illustration. Based on this difference, this feature is used in our scheme to distinguish blur from sharpness.

Because the blurry condition is so localized, obviously the power spectrum is not enough to describe the distinction between blur and sharpness. It is because "a blurred region" is defined by a relative comparison, not resulted from an absolute threshold, hence it is essential to employ localized information when estimating blur. An averaging process is involved here to preserve localities in different pictures for the estimation. The averaging process among image blocks is shown in Equation (8). The blur-sharpness discrimination is done by calculating $S_{N}$. The effect of this normalizing process shown in Fig. 5 (c) could diminish the divergence of signal variation because the power spectrums are normalized, thus which region is blurrier will be clarified. Although the difference could be told between blurrier and sharper, in order to 
separate the boundary of blur and sharpness, a threshold is necessary, see Fig. 6.

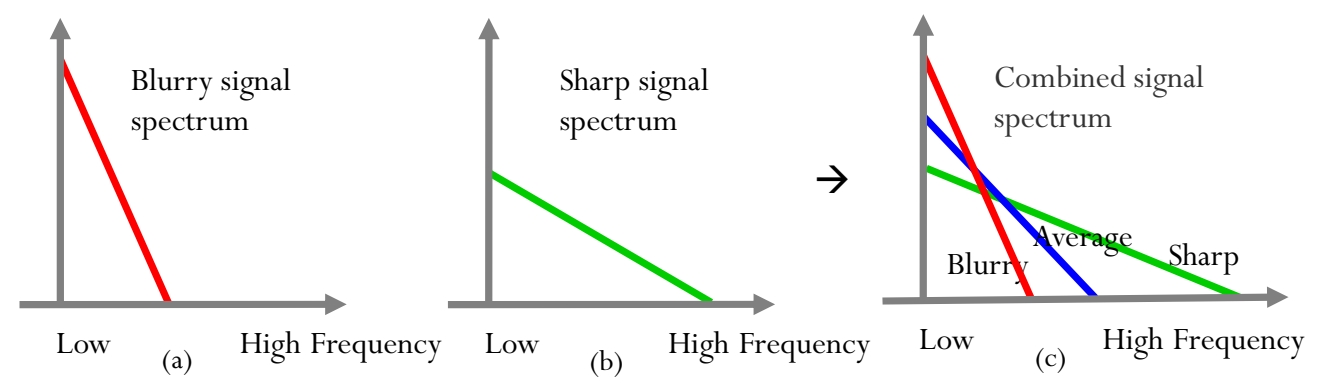

Figure 5. The coarse assumptions on the power spectrum distribution of a blurry signal, a sharp signal, and a power spectrum averaged between blurry signal and a sharp signal.

$$
S=\left(\begin{array}{ccc}
B_{11} & \ldots & B_{1 N} \\
\vdots & \ddots & \vdots \\
B_{M 1} & \ldots & B_{M N}
\end{array}\right), \quad A=\frac{1}{M N} \sum_{i, j} B_{i j}
$$

Where $S$ is the block by block DCT or FFT transformed source image, B is DCT or FFT

transformed $8 \times 8$ block, and $i=1 \ldots M, j=1 \ldots N$

$$
S_{N}=\left(\begin{array}{ccc}
C_{11} & \ldots & C_{1 N} \\
\vdots & \ddots & \vdots \\
C_{M 1} & \cdots & C_{M N}
\end{array}\right), \quad C_{i j}=\left\{\begin{array}{c}
B_{i j} / A, \\
\text { or } \\
B_{i j}-A
\end{array}\right.
$$

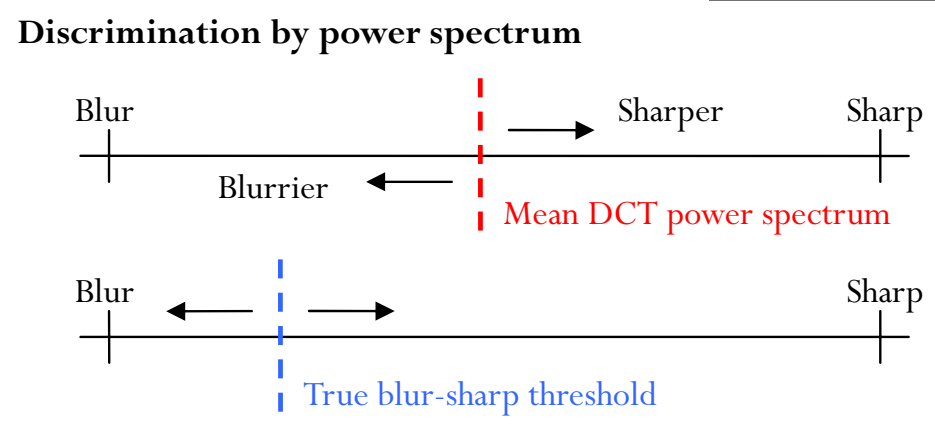

Figure 6 . The figure shows that threshold adjustment is required.

Some tests are performed to verify its effectiveness. Fig. 7 contains a sharp object and also some wood plate textured regions which are in-focus. The blur of this image is resulted from defocus; the other three images represent the predicted sharp regions with three different thresholds; black region denotes sharp area.

Form Fig. 7 shown above, it can be concluded that a precise threshold will generate good blur estimation. We can carry out the whole estimation scheme shown as Fig. 8 and descriptions about each phase of the scheme is presented below.

The first phase provides adaptive adjustment of threshold, or prior guess about the blur-sharp threshold. The normalized DCT coefficients in the second phase could offer the information about what regions are blurrier or sharper compared to the whole image, but not provide the 
true boundaries and indicates where the blurred regions are or there are no blurred regions at all, thus we need a threshold here to make the final decision about what region should be labeled as a blur. If the threshold mechanism does not exist, in other words, the threshold is a constant value; inevitably, an entirely sharp image will be declared as partially blurred and same will happen in an overly blurred picture.
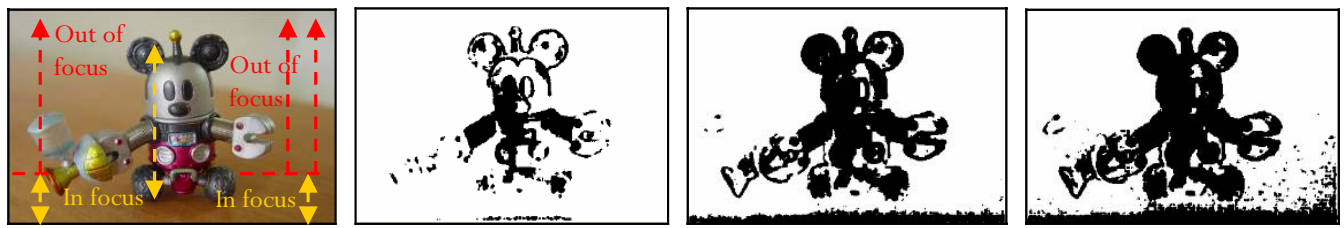

Figure 7. The figures show that if the threshold is selected properly, the result may match the observation (the toy, and some of the ground in-focus). Because the contents and scene composition, clearly that the threshold will be specific to each image.

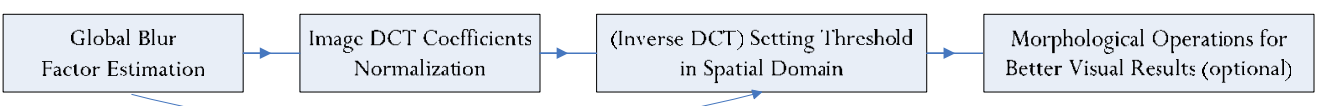

Figure 8. The system flow of the blur estimation algorithm

In order to obtain accurate blur-sharpness threshold, we apply a method similar to [1] but with some differences. Based on its blur determination method, a quality factor of blur percentage is quantified, and this factor is used as an index in setting our blur-sharpness threshold. With this prior information, we could first detect the image is globally blurred or not then we adjust our threshold to meet the condition of the target image. For example, a blur factor of $98 \%$ indicates a really sharp image, and the threshold must be high to ensure most parts of the image are labeled as sharp ones. In the final phase, with this measurement, we can obtain good and accurate estimation even the image is extremely blurred or sharp.
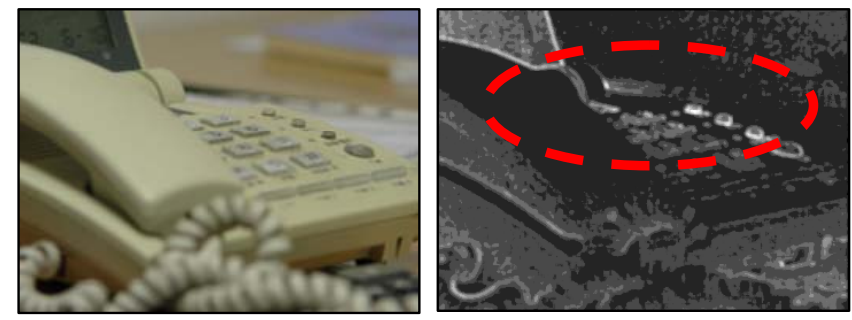

Figure 9. The above images are used to show the result of the power spectrum discrimination method by comparing power spectrums of each block to the average power spectrum of the image, mentioned as the second phase. The high intensity regions (brighter white blobs) marked by the circle represent the sharper regions and the outcome is consistent with the input image. The low intensity regions (darker ones) indicate blurrier areas.

The second phase is the blur estimation core of the whole process. A mean DCT block of coefficients of the target image is calculated in this phase. Then we process through every DCT block to normalize to this mean DCT coefficient block. Taking the mean DCT coefficients as a normalization threshold is better because the mean values are more content dependent thus this 
separation is more accurate and independent of contents. After normalization, we convert the image into spatial domain and smooth it to reduce the edginess of the resulting image. Now we have an overview about the image of its blur condition, take Fig.9 for example: brighter parts of the image indicate sharper regions, and the sharpest areas are extremely illuminated; equivalently, darker parts of the image are in the sense of blurred regions or smooth surfaces.

$$
\text { where }\left\{\begin{array}{c}
C_{i j}=B_{i j} / A, \text { or } \\
C_{i j}=B_{i j}-A
\end{array}\right.
$$

The third phase is done by varying thresholds from the interval to four times of standard deviation, plus or minus. The amount of threshold is adjusted with the quality factor acquired from the first phase. There is a mapping function that maps the value of the quality factor to the corresponding threshold. However, this mapping function has to be trained manually once; by recording an image's quality factor and listing the threshold that will generate the desired result, and then using polynomial approximation to obtain a mapping curve. With such a mechanism, the threshold will be more precise and consequently gain a better result. Blurred regions are shown as white regions in the detection results. In the case that uses FFT for the automatic blur estimation, we obtain a training curve, which is approximated to the $3^{\text {rd }}$ order, is shown below:

$$
\begin{aligned}
& \text { Prior Knowledge Factor }= \\
& -319.61 \times(\text { quality factor })^{3}+843.98 \times(\text { quality factor })^{2}-744.88 \times(\text { quality factor })+219.51 \\
& \text { Threshold }=\text { mean }+(\text { prior knowledge factor }) \times 4 \times(\text { standard deviation })
\end{aligned}
$$

Where the prior knowledge factor is in the range of [-1,1], if prior knowledge factor is out of the range, or saturated, it will be set as -1 or 1 .

The optional phase is doing opening and closing morphology to generate more solid results. Some results will be demonstrated the effectiveness of blur estimation in the result part in this chapter, and the proposed scheme will show that it provides good estimates in both naturally blurring and manually smoothing on a single image. However, when blur estimation algorithm is applied for digital tampering detection, some modifications are necessary. The system flow of the original blur estimation is adjusted to fit the requirement of digital fraud identification.

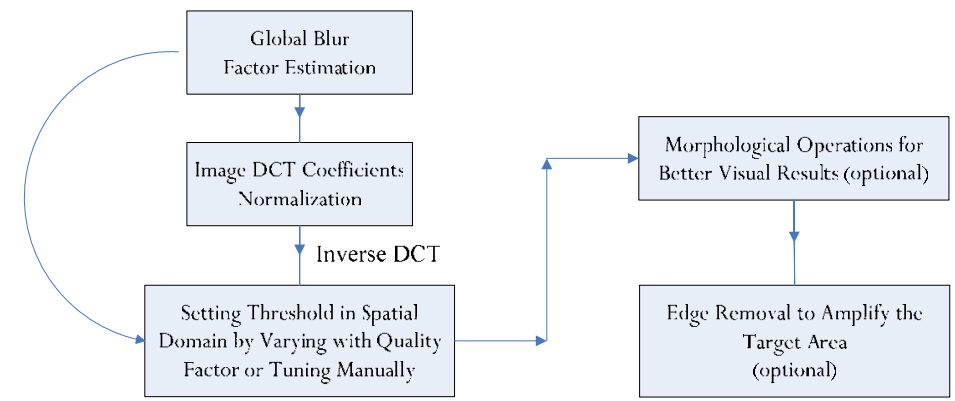

Figure 10. The modified system flow of blur estimation in digital forgeries.

In the modified scheme for digital forensic detection, we add optional procedures such as image enhancement and edge removal to obtain more robust outcomes. What is more, considering the practical situation, to make a fraudulent image, one usually changes only a few parts of the image rather than alters the entire image. So it is very likely that an image accompanied with some blurry regions is used as the original but these blurred regions remain unaltered after the tampering is done; thus straightforward blurred region detection will not provide direct answer to the tampered area, imaginable, it will report other blurred regions in the image, too. 
Although having this difficulty, other than the automatic detection, we add a fine tuning for the detection, giving us the chance to recalibrate the threshold and see if there are blurred regions appeared oddly in focused regions. In this way, odd blur appearing could indicate the existence of possible tampering by restricting weird blur shows in the target area. The detection results are present in the result section.

\subsection{Digital image tampering detection using blur estimation}

From the knowledge of blurring characterized by point spread function, we know that if we restrict the spectrum for only a certain kind of signal (or texture), the relationships (sharp and blurred ones) will match the illustration as Fig. 5, which means we could estimate a small or a large amount of blur, resulted from a small or a large deviation from the focus plane of the subject. Seeing that the blur estimation will give us some clue of depth, and with the result of blur estimation applied on normal images using various thresholds, it becomes possible to construct a valid tool for blurring involved digital image fraud detection.

The main idea we use to expose digital image blurring is by examining the consistency between defocus knowledge (depth of field) and blur estimation results (blurrier regions). For example, in Fig. 14, we know that the focus is on the boy thus he should be a sharp object, and so is his entire face because they are all in the focus plane, this is called the defocus knowledge. If the blur estimation results show that some part of his face disobey the defocus knowledge, there must be some other procedures like blurring is taken on the image; in this example, the blurring applied on his cheeks and chin is revealed.

Note that other than examining the inconsistency between depth of field and results of blur estimation, we could define some region is highly potential for digital tampering. Considering the face replacement case, the cheeks are usually the location smoothing will be applied on. If we could observe odd blur appearance on such region, we can say the image is suspicious for being manipulated.

\section{Detection results}

We have test on several kinds of images, the following results are grouped by the manner of test images. We will first present the effectiveness of blur estimation on unaltered images, then the demonstration about the frequency domain approach of blur estimation to identify the existence of possible tampering.

In the first group, results are the of normal photography image. We test on the image which has partial sharp and blurred regions. As seen in Fig. 11 (a) is the input image, which has a quality factor of $78.07 \%$; this number is generated from the first phase, indicates the degree of global sharpness. Fig. 11 (b) is the normalized image of Fig. 11 (a), which is the result of the second phase. It could be observed that sharper regions are brighter and blurrier regions are darker. After threshold applied, final result is shown in Fig. 11 (c), black blobs indicate sharp regions and white blobs indicate blurred ones. This algorithm does indicate the sharp regions (keypad on the phone) and blurred ones (backgrounds). Blurred regions are locally estimated, blur percentage is $88.3848 \%$. The final results and are quite close to our expectation.

Since the algorithm has shown its effectiveness in detecting localized blur on normal images, we furthermore verify our method on some other extreme sharp/blurred cases. Fig. 12 represents the cases of overall sharp/blurred images. If the threshold is not taken adaptively, we will expect some blurred region detection in an overall sharp image or some sharp region detection in an overall blurred image. Our method could avoid those misdetections via the global sharp/blur degree estimation. The quality number is $100 \%$ in (a), which means a tight 
threshold is applied to this image; hence the blur detection is $0 \%$ (e). On the other hand, the image of (b) has a quality number of $73.75 \%$, which will result in a loose threshold on this image; the blur detection result is $100 \%$ blurred. In these cases, extreme sharp/blurred images are detected correctly, and the essentiality of the first phase is unfolded.

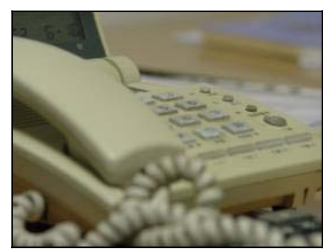

(a)

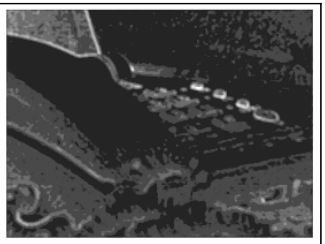

(b)

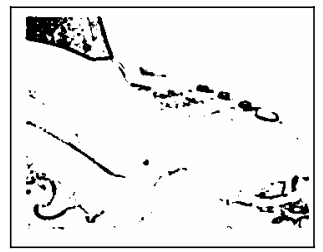

(c)

Figure 11. Local blur estimation on a partially defocus blurred image, (a) a partially defocus blurred image, (b) the blur estimation result of (a) after the second phase, with brighter blobs indicating sharper regions while darker blobs indicating blurrier regions, (c) the final blur estimation result of (a), with white blobs indicating blurred regions.

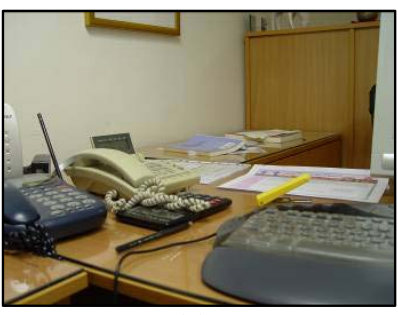

(a)

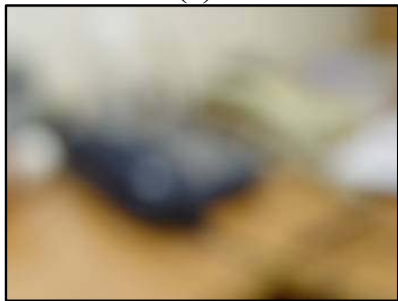

(b)

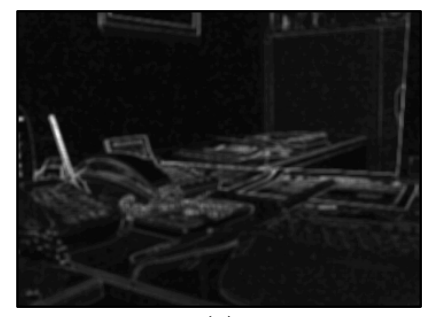

(c)

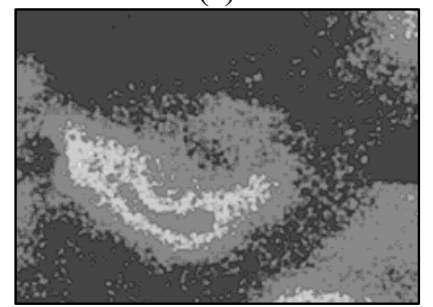

(d)

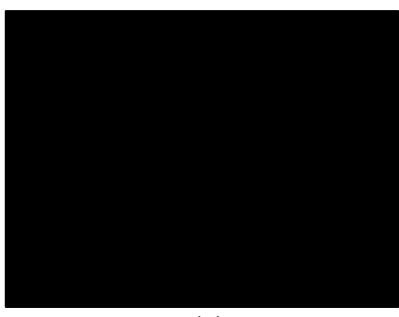

(e)

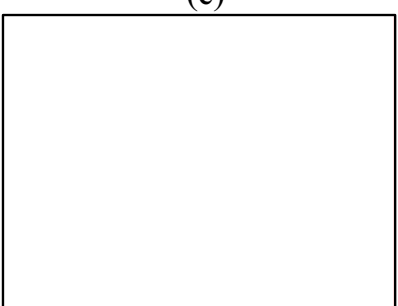

(f)

Figure 12. Comparison of local blur estimation between overly sharp and blurred images, (a) an overly sharp image, (b) an overly blurred image, (c) the blur estimation result of (a) after the second phase, with brighter blobs indicating sharper regions while darker blobs indicating blurrier regions, (d) the blur estimation result of (b) after the second phase, (e) the final blur estimation result of (a), with white blobs indicating blurred regions, (f) the final blur estimation result of (b).

After those experiments on images which are blurred by defocus, we perform our test on those images blurred by Gaussian filtering. The test image Fig. 13 is the texture from ordinary floor (shot is taken perpendicularly to the floor), and the image is applied with Gaussian blur of 4 pixels on its right half. The result of Fig. 13 is clear. From above results, it could be shown that our proposed scheme is effective in localized blur detection and most outcomes it offers are reliable. 

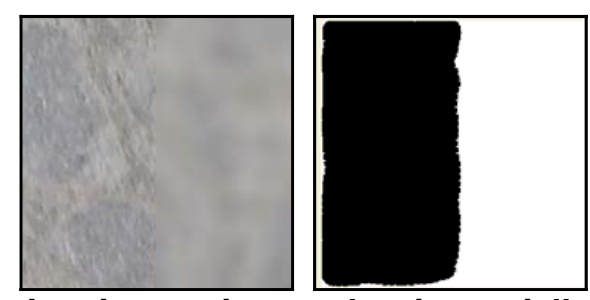

Figure 13. Local blur estimation on image that is partially blurred manually, left, the original input, blurred partially at the right half. Right, the blur estimation result, indicates the half blurred area with minor estimation error on the edge.

The result applying blur estimation on digital blurring involved tampering are presented as following groups. The first demonstration that Fig.14 shows is to detect skin smoothing traces; skin smoothing is applied very frequently in digital photography post-processing. In the result image, the regions of the boy's cheeks and chin are represented in white blobs (blurred areas), whereas such phenomena disobeys the defocus condition in the whole image. Such an inconsistency implies that smoothing operation is taken.

The subject of Fig.15 is ordinary face replacement problem. In this case, we crop areas estimated as blurred. Because of the smoothing in face replacement, the entire forged face is verified as blurred, and the inconsistency between anticipated defocus blur and the estimation result indicates possible presence of face replacement operation. In Fig. 16 and Fig. 17, the blur estimation reveals blur in the highly suspicious region (cheek), and this meets the second idea that we identify digital tampering. Comparisons between original and tampered are given to verify such an idea.
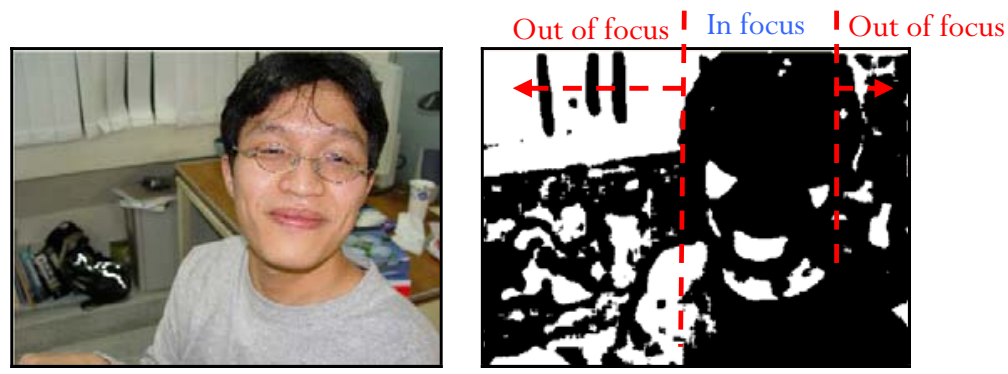

Figure 14. Left, the original, the boy's cheeks and chin are smoothed; right, blur estimation result. Note that other than the defocus regions, the boy's cheeks and chin are also labeled as blurred, which is extraordinary since all in-focus regions (other regions of the face, part of the clothes) are classified as sharp.
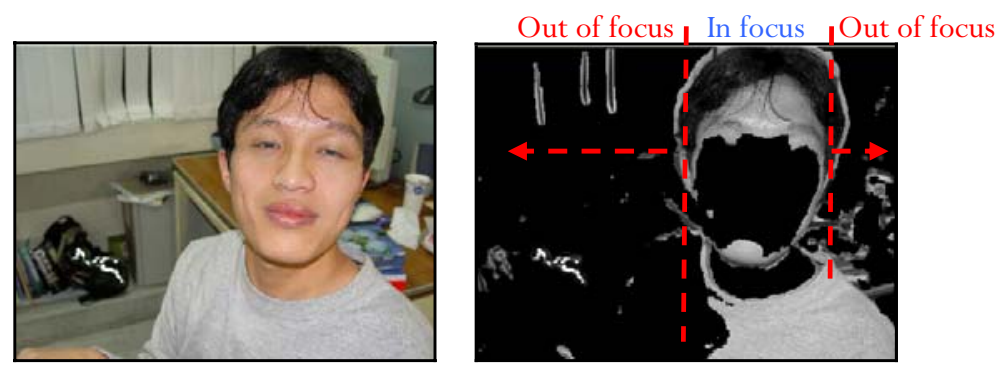

Figure 15. Blur estimation result on face replacement, which displays the possible in-focus parts of the image and dismisses the defocus regions. The result implies that there is potential of smoothing operation performed on the face of the boy. 

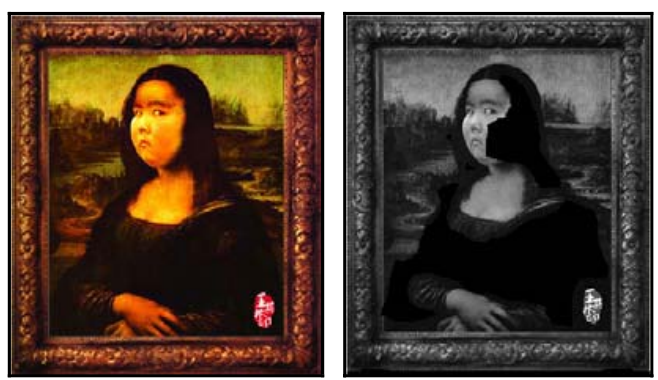

Figure 16. This is another example of face replacement, note that in the result image, the cheek of the face is cropped, which implies potential possibilities of tampering, in this example, it is a smoothing process.
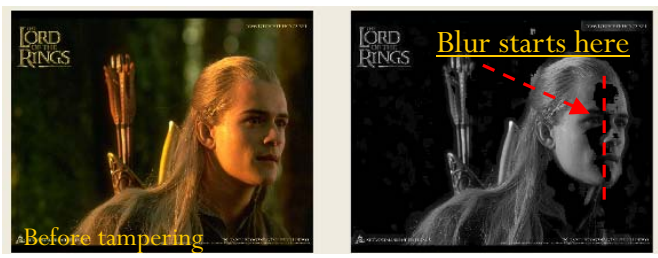

(a)

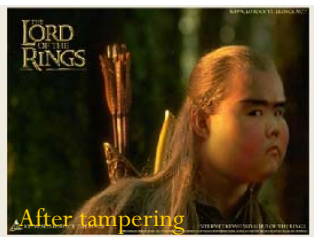

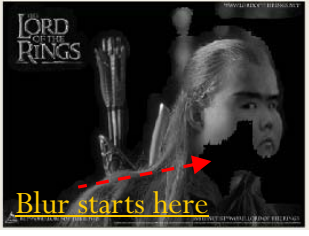

(b)

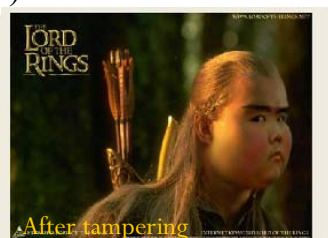

(c)

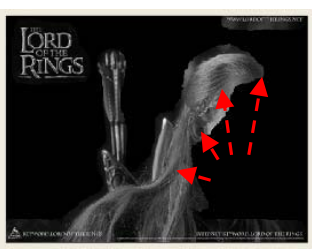

Figure 17. Test to verify the existence of difference of the blur estimation results between the original image and the face-replaced forgeries.

In Fig. 17, the original image (a), its estimated region of possible blur is shown at the out-of-focus background and some part on the man's face, starting from the area that next to his nose, in the shadow. But in the face-replaced ones Fig. 17 (b) we couldn't see such phenomena, the estimated blurred region starts from the chin and cheek, which are the place that have highly potential to perform a smoothing operation in order to remove visible edges resulted from face replacement. In Fig. 17 (c) it is done by adjusting the threshold of Fig. 17 (b), the blur estimated regions grow all over the face, leaving the unaltered region (original) only.

Among the results, we discovered that blur estimation also provide us some useful information about the depth composition of the objects in the image. Because objects may not share the same focus plane, they would have different amount of blur from defocusing. We could trace the consistence in the relation of blur amount and object depth to resolve splicing forgery problems. Such as the result group of Fig. 18 shows, the two objects have been spliced together and share the same focus plane, but they have different amount of intrinsic sharpness which is not perceptible for human while blur estimation would separate them respectively. 

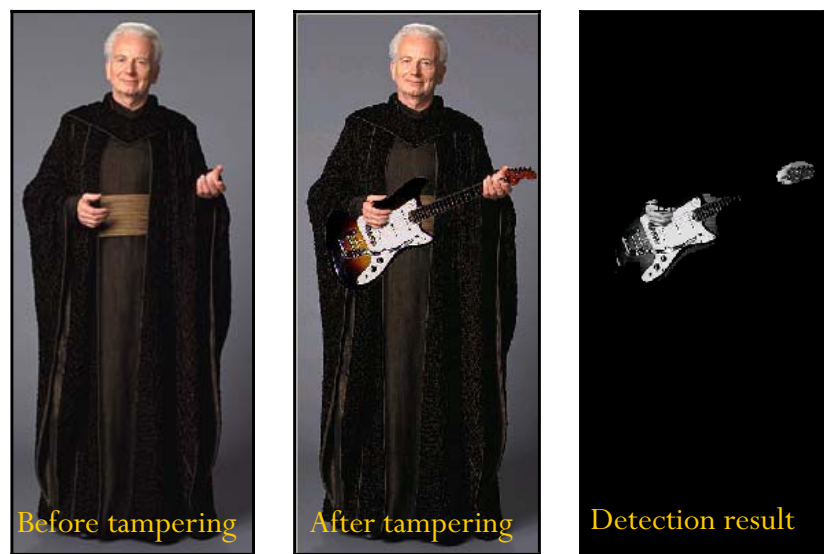

Figure 18. From the correlation of depth and blur, in this image, although it is made by splicing the guitar image to the original, doing blur estimation also exposes the inconsistent of the blur amount corresponding to its depth; the man is in the same focus plane with the guitar, but apparently they exhibit different amount of sharpness and leave the trace for us.

\section{Detecting computer-generated graphics}

Even we have several magnificent computer graphics techniques today, some odd characteristics resulted from the virtually composed objects could not hide from the observation of human eyes. However, if the virtual objects are inserted in fast motion scenes, they are often unnoticed by viewers because of human vision limitations. However, when we bring the motion pictures to still images, it is possible to reveal those plausible entities. To prove this idea, we present some examples for demonstration, once again, we use blur estimation as our tool.

\subsection{Computer-generated object detection using blur estimation}

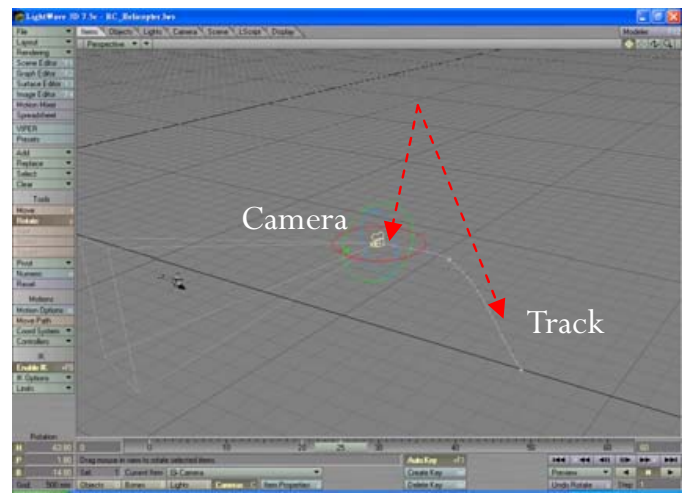

Figure 19. A snapshot from the generating stage of a CGed scene, the camera and the track are shown in the canvas. Note that the camera is set to have a track to shoot the image, which is very common in animation production. Since the track is predefined, there will be no jitter at all, and different than shooting of real films.

In rendering computer-generated objects, the position and motions of the characters and the camera are settled before rendering, so there will be no jitters, no aberrance, only regulations 
and routines; the rendering result will be carried out as predicted and as it was set to be.

However, in a real shooting stage, the screen is captured by a camera. Although in a motionless shot, no matter the camera is doing a still shot or following the subject coherently, in either way it will produce a small quantity of motion blur resulting from camera jittering. In a motion shot, the entire screen is of course composed of global motion blur produced by camera movement and local motion blurs resulted from the moving objects. This motion blur feature is observable in general real movie scenes.

Based on the two difference image producing methods, we could verify the blur condition of the image to see whether it contains computer-generated objects or not. For example, in a real scene, the object will have a large motion blur when it is in motion, and it will still have some minor motion blur even motionless, such those in Fig. 20; but a computer-generated object won't have such a feature, it will exhibit very sharp textures, for instance, Fig. 21. In Fig. 20, the left image shows a small quantity of motion blur because the horsemen are moving slowly referred to the camera. Whereas right one is bit different, with greater motion (riding the horse) but a partially sharp region (the face of the king) because the camera is following the actor's face. However, the face is still a little bit blurred because the actor is not coherent exactly to the camera he faced. As it is predicted, the some odd motion blur will appear. Referring to Fig. 21, the mammoth group is moving in rapid and large movements. They swing their ivories, but such movements form an unnatural motion blur: some part of the object (some regions on the mammoth's forehead) still have very sharp textures and several neat edges. This phenomena is inconsistent with the real motion scenes, considering Fig. 22 (a), the real scene image is totally blurred in motion. Such difference in the expression of motion blur makes it a possible way to distinguish the computer-generated from original counterparts (real horsemen, soldiers) in those snapshots. The snapshots are taken from The Lord of the Rings: The Return of the King.
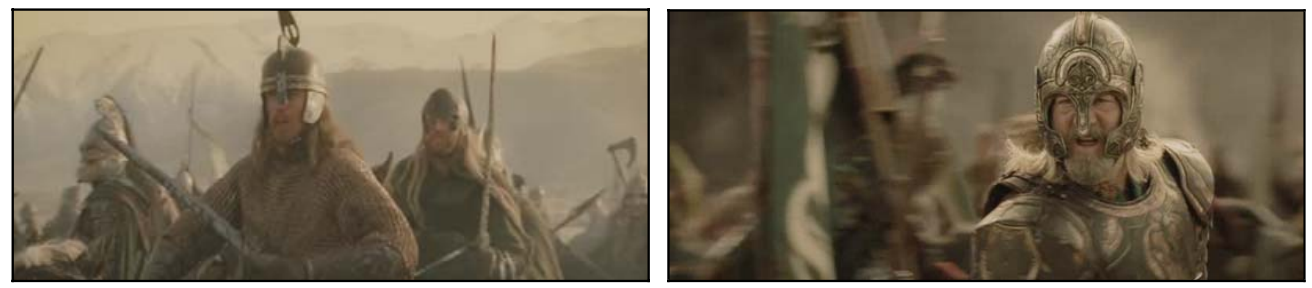

Figure 20. Real object motionless and motion shots.
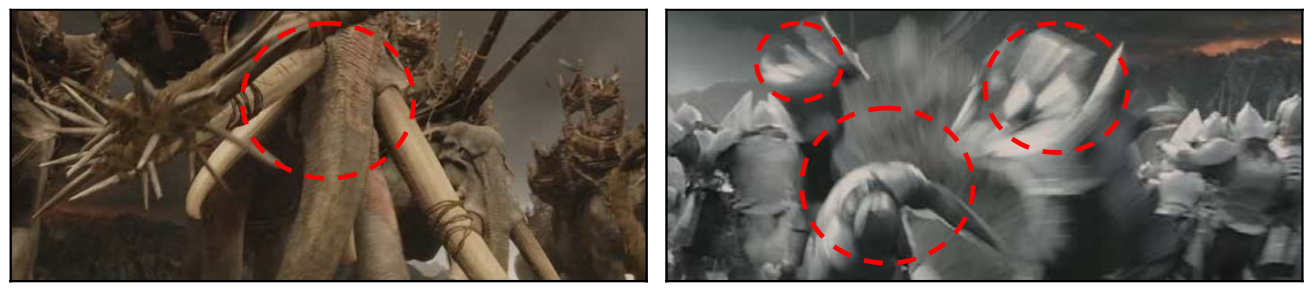

Figure 21. Computer-generated objects motion shots.

Images in Fig. 22 are several battle scenes in the movie, Fig. 22 (a) is totally blurred by motion, and the favorable result is overall in white. Fig. 22 (b) is another which contains a sharper region (the face of the king), other parts of the image are totally blurred by motion. However, the region should still suffer from a minor motion blur because of camera jittering. Its blur estimation shows the face is still in a blur, a global blur of real scenes, as predicted. In Fig. 22 (c), odd sharp edges are appeared on digitally created and blurred soldiers. Blur estimation result of this image reveals the unnatural sharp edges on the digital moving soldiers. 

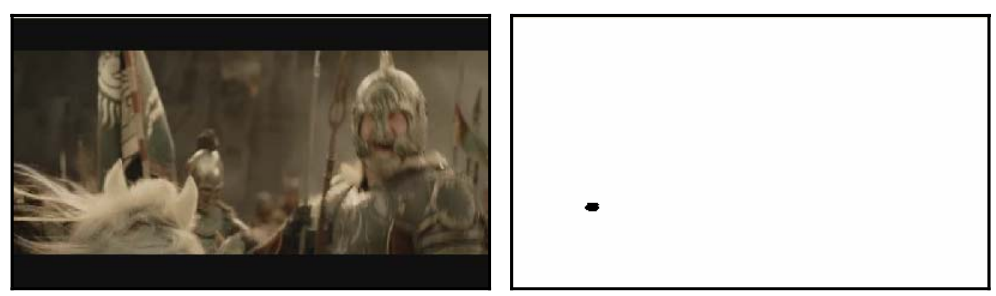

(a)
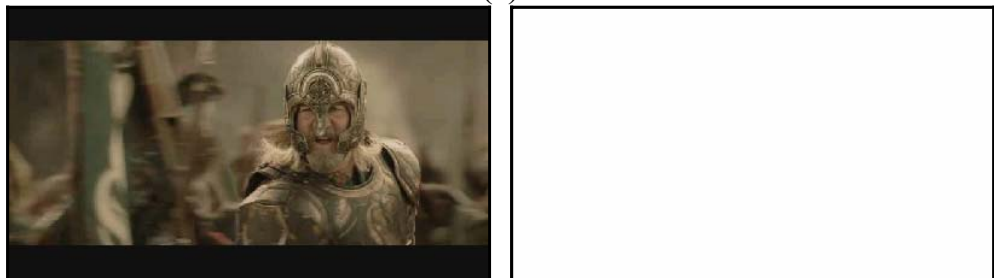

(b)
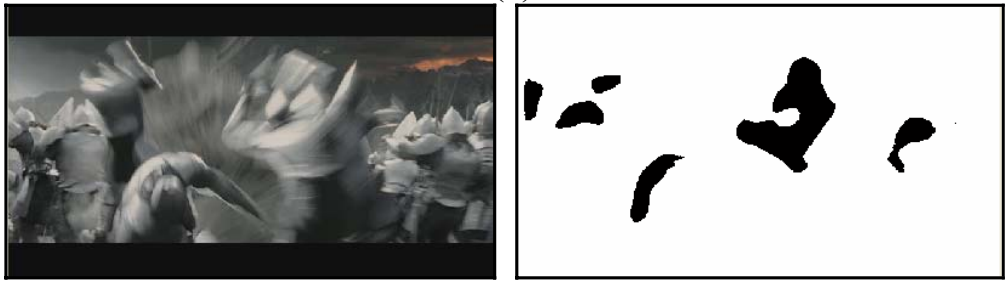

(c)

Figure 22. Blur estimation in real scenes (a) (b) and computer-generated object inserted scene (c), some very sharp regions are verified in (c), as black blobs.

The above results shown here provide the evidence that it is possible to trace virtual object in a composite scene. Although it is likely to have features different from one movie to another, the motion blur variation is based on a more general assumption, and offers a better possibility to have a successful detection. Blur estimation has proved proper to estimate motion blur and help determine if the blur is globally or locally, and the outcome generated is quite anticipating.

\section{Discussions}

The first problem is about the dimension of the subject source. When the source shrinks its size, it means some information is abandoned or merged like a multiple to one mapping. In this way, it will reduce useful features in the source for us to detect. For example, considering the case that splicing two images, one could first up-sample the image to a larger one for convenience to cut the desired part, and often the edges are not cropped neatly; however, one may remove the visible unwanted edged by resize the spliced image back to the original size or a even smaller one. Another tough problem is about the compression, compression will provide a great amount in the reduction of redundancies, but it also discards imperceptible high frequencies which are essential to the detection of blur estimation. Blur estimation rely on the assumed noise to construct the distribution of the power spectrum or show the distinct pattern of tampered regions. A JPEG compression, if applied to compress the source hugely, will have the same effect of blurring and confuse and even eclipse the result of estimation.

Lucklessly, such problems always occur in every kind of digital frauds detection. Moreover, there are potential possibilities that the source has the same feature of the fraud originally. One example is the face replacement on movie posters; the movie posters usually cast smoothing on 
the skin of its character or the whole scene to make a dramatic effect, but this will obscure the observation after blur estimation is applied, we will have no clue about which are originally blurred and which are not. But successfully with the assistance of manually adjustment, we could verify some difference between originally blended regions and additionally cast ones.

Although having such a difficulty, the blur estimation exposes potential blurring related digital image tampering effectively.

\section{Conclusions}

We proposed a method that is able to detect blurred regions in an image. And the results of the detection show the method is reliable in various kinds of images even there are no blur in the image at all or the blur is made artificially. From the idea that blending is inevitable to hide unwanted traces in the making of digital forensics, we applied modified approach of blur estimation based on frequency domain knowledge.

The effectiveness of blur estimation on plausible digital image frauds, even on those non-perceptible ones for human are demonstrated. By combining additional information, such as depth relationships of the objects or unnatural sharpness, both help identify digital image frauds. All tests of this method are operated on a computer environment of $1.3 \mathrm{G} \mathrm{CPU}$ with 640MB RAM. For each run of estimation, the calculation interval depends on the size of the target image and varies from couples of seconds ( 5 mega pixels image) to instant. Experiment results show that this scheme is powerful in blurred regions detection for defocused images as well as digital image tampering involving blurring process, with the advantage of simplicity.

\section{References}

[1] Marichal, X.; Ma W. Y.; Zhang H. J.; "Blur determination in the compressed domain using DCT information" in Proceedings of International Conference on Image Processing, vol. 2, pp. 386 - 390, 1999

[2] Elder, J. H.; Zucker, S.W.; "Local scale control for edge detection and blur estimation" in IEEE Transactions on Pattern Analysis and Machine Intelligence, vol. 20, No. 7, pp. 699 - 716, 1998

[3] Elder, J. H.; Zucker, S.W.; "Scale space localization, blur, and contour-based image coding" in Proceedings of IEEE Computer Society Conference on Computer Vision and Pattern Recognition, pp. 27 - 34, 1996

[4] Popescu, A.C.; Farid, H.; "Exposing digital forgeries by detecting traces of re-sampling" in IEEE Transactions on Acoustics, Speech, and Signal Processing, vol. 53, No. 2, pp. $758-767,2005$

[5] Lyu, S.; Farid, H.; "How realistic is photorealistic?" in IEEE Transactions on Acoustics, Speech, and Signal Processing, vol. 53, No. 2, pp. $845-850,2005$

[6] Popescu, A.C.; Farid, H.; "Statistical Tools for Digital Forensics", Department of Computer Science, Dartmouth College

[7] Lyu, S.; Farid, H.; "Higher-order Wavelet Statistics and their Application to Digital Forensics" in IEEE Workshop on Statistical Analysis in Computer Vision, 2003

[8] Farid, H.; "Detecting Digital Forgeries Using Bispectral Analysis", Perceptual Science Group, MIT, Cambridge, MA 02139

[9] Popescu, A.C.; Farid, H.; "Exposing Digital Forgeries by Detecting Duplicated Image Regions", Department of Computer Science, Dartmouth College

[10] Popescu, A.C.; Farid, H.; "Exposing Digital Forgeries in Color Filter Array Interpolated Images" , Department of Computer Science, Dartmouth College

[11] Ng, T. T.; Chang, S. F.; "A model for image splicing" in IEEE International Conference on Image Processing, vol. 2, pp. $1169-1172,2004$

[12] Ng, T. T.; Chang, S. F.; Sun, Q.; "Blind detection of photomontage using higher order statistics" in IEEE Proceedings of International Symposium on Circuits and Systems, vol.5, pp. 688 - 691, 2004

[13] Ng, T. T.; Chang, S. F.; "A Data Set of Authentic and Spliced Image Blocks" in ADVENT Technical Report, No. 203-2004-3, Columbia University, Electrical Engineering Department, Columbia University, New York, 2004

[14] Ng, T. T.; Chang, S. F.; "Blind Detection of Digital Photomontage using Higher Order Statistics " in ADVENT Technical Report, No. 203-2004-3, Electrical Engineering Department, Columbia University, New York, 2004

[15] Fridrich, J.; Soukal, D.; Lukás, J.; "Detection of Copy-Move Forgery in Digital Images", Department of Electrical and Computer Engineering, Department of Computer Science

[16] Kerlow, I. V.; The Art of 3-D Computer Animation and Effects, $3^{\text {rd }}$ ed., John Wiley, ISBN: 0471430366 